\title{
Colliding heavy ions: Nuclei as dynamical fluids ${ }^{\star \dagger}$
}

\author{
James J. Griffin ${ }^{\ddagger}$ \\ Department of Physics and Astronomy, University of Maryland, College Park, Maryland 20742 \\ Kit-Keung Kan \\ Department of Physics and Astronomy, University of Maryland, College Park, Maryland 20742 \\ and Department of Physics, University of Texas, Austin, Texas 78712
}

\begin{abstract}
Heavy ion experiments are already enriching nuclear science with a tapestry of new phenomena which require explanation. In response, theoretical nuclear physics is rapidly expanding its insights to encompass these new observations, especially those concerned with the macroscopic aspects. Preliminary theoretical studies already suggest that the dynamical nuclear fluid must sometimes be considered viscous, ${ }^{1}$ compressible, and/or rotational, if its microscopic properties are to be encompassed. These and some threads already well placed in the picture will be discussed. Other reasons will be cited to support the expectation that theoretical nulear macroscopists may more and more come to be fluid dynamicists who specialize in those few thousand fluids called nuclei. Three such reasons are (a) the promised richness of their structure as dynamical fluids, (b) their unique prospect, among all the objects of modern physical science, of allowing a complete microscopic, as well as a phenomenological macroscopic, description, and (c) the possible overflow of such nuclear implications into classical fluid theory, from the viewpoint of which the nuclear heavy ion data are a significant novelty.
\end{abstract}

\section{CONTENTS}

I. Data, Parameters, and the Hydrodynamical Analogy

A. Heavy ion data is rich and complex

B. Some qualitative features still need explanation

C. Frictional forces are useful in describing heavy ion collisions

D. Colliding water droplets may teach us about nuclei

II. Fluid Dynamics in Schrödinger Theory,

Compressibility and Inertia

A. Fluid dynamical concepts and Schrödinger theory

B. Dynamical compressibility

C. The effect of compressible flow on inertial parameters

D. Analogy with Strutinsky shells

E. Every nucleus a fluid of its own

III. Novel View of Quantum Physics via the Single-

Particle Schrödinger Fluid

A. Irrotational velocity

B. Regular velocity

C. Collective kinetic energy

D. Single-particle Schrödinger fluid: Some details

IV. Quantum Friction-One Spinoff

V. Acknowledgments

Refer ences
467

467

467

468

469

469

470 470

470

471

471

471

472

472

472

473

474

476

476
*This paper is adapted from an invited talk given by J. J. Griffin at the Pittsburgh Meeting of the Division of Nuclear Physics, American Physical Society, on October 31, 1974 (Bull. Am. Phys. Soc. II, 19, 996).

†Work supported by U. S. Energy Research and Development Administration.

+Present address: Institut für Theoretische Physik, Universität Giessen, Giessen, Germany, and the Hahn-MeitnerInstitut für Kernfor schung, Berlin, Germany. The support of the Alexander von Humboldt Foundation during the 1975-76 year is gratefully acknowledged.

${ }^{1}$ In retrospect, the word "dissipative" might better have been used here to avoid any suggestion that the microscopic structure of the dissipative process (so far unknown) is necessarily associated with the particular "hydrodynamical" form familiar from the Navier-Stokes equation.

\section{DATA, PARAMETERS, AND THE HYDRODYNAMICAL ANALOGY}

This paper attempts to communicate a few simple ideas on the general subject of nuclei as dynamical fluids. It is in no sense a "review" of the subject, which is probably too new anyway for cataloging, but rather an outline of one still changing viewpoint, and of some recent related research.

The first section of the paper attempts to demonstrate for those who are unfamiliar with recent heavy ion studies that heavy ion data is rich and complex. It also suggests the view that the utility of fluid dynamical parameters is already well established in organizing the phenomenology of heavy ion collisions. As an illustration, we emphasize the idea that macroscopic liquid droplets in collision are very interesting bases for gaining insight into nuclear collision.

\section{A. Heavy ion data is rich and complex}

Figure 1 is taken from Kratz et al. (1974). It presents the results of their radiochemical studies of the products from ${ }^{84} \mathrm{Kr}$ accelerated against ${ }^{238} \mathrm{U}$ at a laboratory energy of $605 \mathrm{MeV}$. (We emphasize that this data is thick target data so that reactions occur over a range of energies, and that radiochemistry measures only those products which live for a substantial time after the collision. Both of these limitations to the information available in this data should be kept in mind.)

\section{B. Some qualitative features still need explanation}

Of richness, Figure 1 is a remarkable example. In the lower half of the figure the several components-one might more precisely say, "Components as interpreted"-in the distribution are labeled by letters A, B, C, D, E, F, G.

Component $\mathrm{A}$ is a broad distribution of the type which is expected to follow the formation of a compound nucleus (the "complete fusion" process) by the amalgamation of the krypton nucleus with a uranium nucleus into 
a single compound nucleus. At the other extreme, the "rabbit ear" distributions, E and F, seem to describe the mass-elastic or nearly mass-elastic processes involving only one, two, or at most a few nucleons transferred, which occur in this reaction. Distribution $\mathrm{C}$ is an example of the "deep inelastic" or "quasifission" process in which a substantial number of nucleons are transferred but still a small fraction of the number (84) in the lighter colliding nucleus, so that the basic identity of the incoming projectile is maintained. The corresponding deep inelastic distribution in the mass region around the uranium rabbit ear $\mathrm{F}$ does not appear, because, one presumes, fissionability of nuclei in that region is very high, especially when they possess a substantial amount of excitation energy, such as a deep inelastic collision would impart. Distribution B is indeed an asymmetric fission mass distribution which might arise from the fission of elements around uranium, and distribution $\mathrm{D}$ is a symmetric distribution which could easily correspond to fission of more highly excited elements. We note that its center is displaced downward, as might naturally be expected to follow from the boilout of some neutrons before the average fission process occurred.

Finally the most remarkable distribution is that labeled $\mathrm{G}$, which seems to peak around mass 200. This is the so-called "Goldfinger," a wholly unexpected discovery of this radiochemical study. It represents a feature of this reaction which has yet to receive an adequate and/or compelling explanation [but, cf., recent discussions by Huizenga (1975), and Griffin and Wong (1975)].

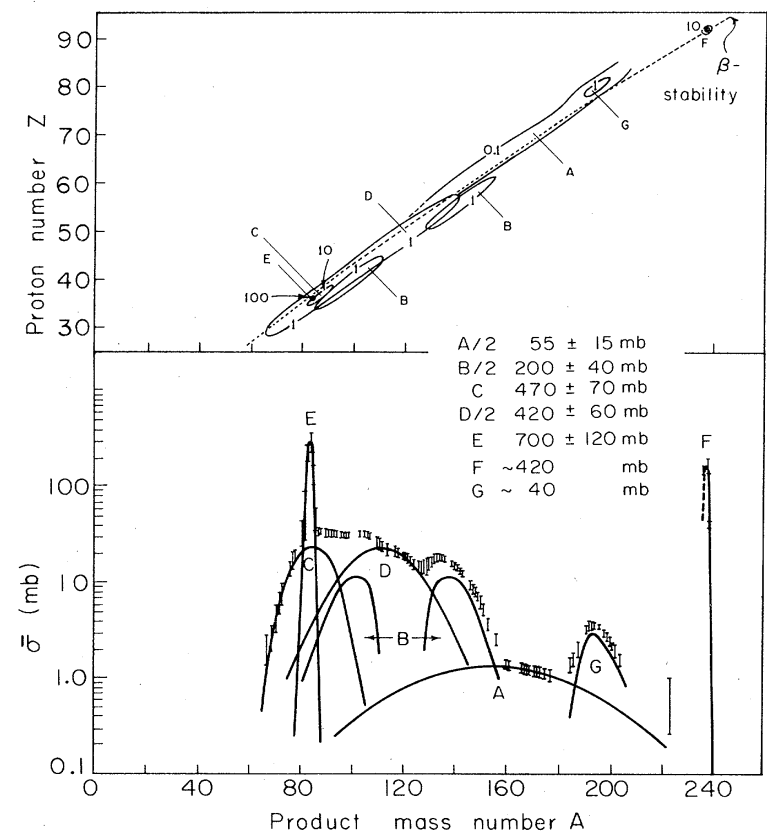

FIG. 1. The (radiochemical) mass and charge yields measured by Kratz, Norris and Seaborg, after the reaction ${ }^{84} \mathrm{Kr}+{ }^{238} \mathrm{U}$ are shown (Kratz et al., 1974). The cross section vs $A$ is discussed at length in the text.

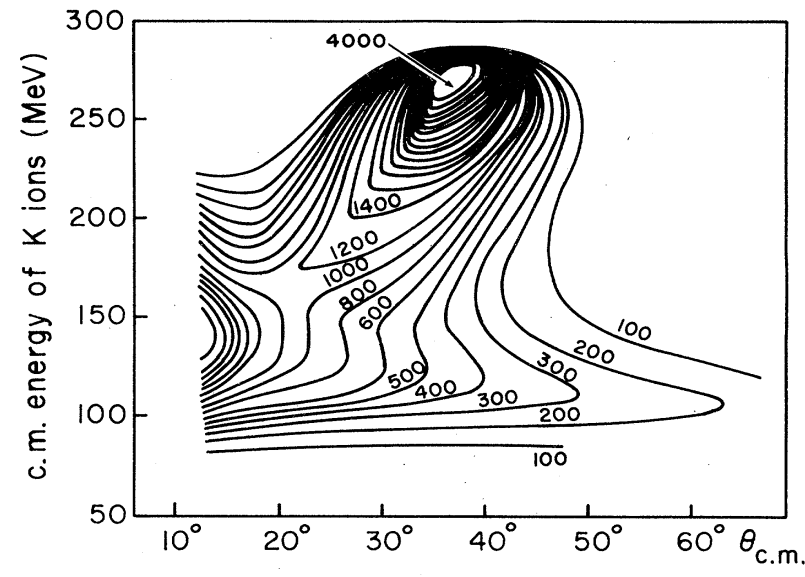

FIG. 2. Equiprobability contours for kinetic energy vs angle of potassium ion in reaction ${ }^{232} \mathrm{Th}\left({ }^{40} \mathrm{~A}, \mathrm{~K}\right)$, from Artukh et al. (1973), after Wilczynski (1973).

\section{Frictional forces are useful in describing heavy ion collisions}

Figures 2 and 3 support the next short statement, that fluid dynamical parameters are useful in phenomenologizing heavy ion reactions. Here we address in particular the energy-momentum dissipation parameter analogous to the hydrodynamical viscosity, and show the data (Artukh et al., 1973) from the reactions ${ }^{232} \mathrm{Th}-$

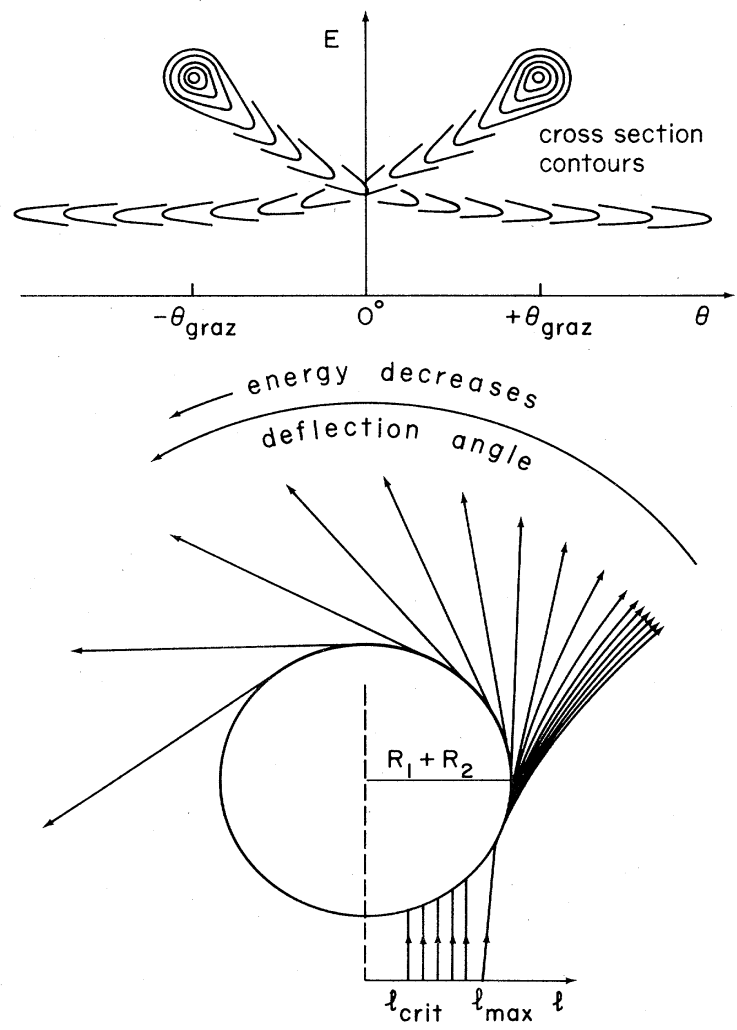

FIG. 3. Wilczynski's interpretation of the data of Fig. 2 (upper part), and his physical picture (lower part) of how a nuclear frictional force can result in such an observation (Wilczynski, 1973). 
$\left({ }^{40} \mathrm{Ar}, \mathrm{K}\right)$ after Wilczynski (1973). One sees that as the scattering angle diminishes from about $40^{\circ}$ toward $10^{\circ}$, the most probable kinetic energy of the potassium ion diminishes very steeply from some $280 \mathrm{MeV}$ to about 100 MeV. In Fig. 3 Wilczynski's (1973) schematization of this same data is reprinted together with his trajectory diagram presenting his (very natural) interpretation of the data: (a) the incoming ion orbits along the periphery of the target nucleus for a time short enough so that its basic identity is unimpaired ${ }^{2}$; (b) the longer the contact time the greater the deviation of the final scattering from the grazing angle. His "frictional" assumption, that the amount of kinetic energy lost increases with longer contact time between the two nuclei, then provides a most natural description of the observed data. It provides also a very persuasive example of the utility of the notion of dissipation in ionion collisions.

\section{Colliding water droplets may teach us about nuclei}

The third remark of this first section is that real hydrodynamical droplets, i.e., macroscopic classical liquid droplets, show promise of providing insight into nuclear heavy ion processes. The data of Dr. Charles Hendricks of the University of Illinois (now at the Lawrence Livermore Laboratory), in the form of moving pictures of controlled collisions of water droplets, illustrate this point very well. Figures 4 and 5 have been sketched to give an impression of Dr. Hendricks' movie-filmed data ${ }^{3}$ for several droplet collisions. [See Adam et al. (1968) for some actual frames.] The movies show examples of fusion reactions, and subsequent oscillations of the amalgamated drops. They also include examples of what we propose to call "necklace" reactions (Fig. 4), in which the drop amalgamates momentarily, oscillates once into a very much flattened shape, then into a long, needle-shaped object, whence it disintegrates into several drops equal in size, sometimes three or four, but sometimes as many as twelve or fifteen. These droplets are about as uniform in size and as well ordered in space as the pearls in a necklace. Hence the suggested name. In the seventh and eighth reactions, the particles come together and then re-fission into two fragments. Finally, the movies show two reactions in which the drops pass one another tangentially, ripping off small droplets from their overlapping edges, in a kind of process we call "peripheral fragmentation," as in Fig. 5.

This data supports the suggestion that the nuclear theorist, in studying these droplet collisions, can strengthen and inform his intuition concerning nuclear processes. Nuclei are, after all, still fluids, we believe, even if a mite too small to see with the camera,

\footnotetext{
${ }^{2}$ Nörenberg proposes that the mass, charge, etc. transfer leading towards equilibrium can be described by a FokkerPlanck-type transport equation (Nörenberg, 1974, 1975).

${ }^{3} \mathrm{Dr}$. Hendricks kindly lent this film to one of the authors (J. J. G.) for presentation at the A. P. S. meeting of October 31,1974 . We wish to express our gratitude for this assistance and to recommend to our readers that here is a case, indeed, where it is better to see the movie than to read the book.
}

VIBRATIONAL INSTABILITY

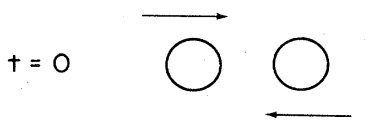

$+\sim 1$

FIG. 4. An artistic impression of the films described in Adam et al. (1968). High velocity head-on collisions lead in one oscillation to a "necklace" of nearly equal spheres. The time unit is $\sim$ milliseconds; the drop radii $\sim 10^{-4} \mathrm{~cm}$.
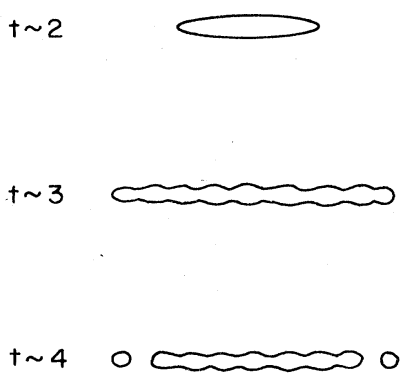

$+\sim 5000000000$

as these classical droplets can be seen. (More about nuclei's own special kind of smallness, associated with their distinctly finite number of nucleons, in sections II.D and II.E below.)

\section{FLUID DYNAMICS IN SCHRÖDINGER THEORY; COMPRESSIBILITY AND INERTIA}

In this second portion of this report, we propose three additional statements: (a) that fluid dynamics fit very

PERIPHERAL FRAGMENTATION

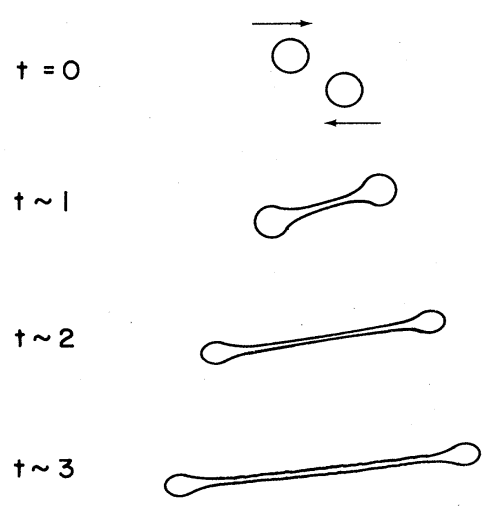

$t \sim 3.500000000000000000$

FIG. 5. An artist's rendition shows how tangential collisions described in Adam et al., (1968) lead to a matter "bridge" which disintegrates into small pieces while the major portion of each droplet continues in a nearly straight line. Time unit and drop radii as in Fig. 4. 
naturally with the Schrödinger theory of quantum mechanics (even leading one to an idealized "single-particle Schrödinger fluid" whose properties will be discussed further below). In addition, (b) the inference is drawn from the study of the single-particle Schrödinger fluid that the nuclei cannot safely be assumed to be dynamically incompressible. More specifically, nuclear inertial parameters can be strongly influenced by the local compressibility of the nuclear mass flow patterns. ${ }^{4}$ Finally, from this discussion, one concludes that (c) for nuclei, large effects on the inertial parameters due to just a few nucleons may require each nucleus to be viewed as a unique and separate dynamical fluid. Then the available nuclear isotopes offer to fluid dynamics some several thousand specific examples against which fluid dynamical theories might be tested, rather than just the single object which each classical fluid constitutes.

\section{A. Fluid dynamical concepts and Schrödinger theory}

We illustrate the first of these ideas by exhibiting ${ }^{5}$ the equivalence of the time-dependent Schrödinger equation with a set of three equations with a specifically fluid dynamical cast which result when one writes $\Psi$, the solution of the Schrödinger equation, in polar form, and separates the equation as follows. In

$$
H \Psi=i \hbar \dot{\Psi},
$$

let

$$
\Psi=\Phi \exp \left\{-\frac{i m}{\hbar} S\right\} ; \quad \Phi \text { and } S, \text { real. }
$$

Then in terms of

$$
\rho=\Phi^{2}
$$

and

$$
\overrightarrow{\mathrm{v}}_{\otimes}=-\vec{\nabla} S,
$$

one obtains the following three equations:

$$
\begin{aligned}
& \vec{\nabla} \cdot\left(\rho \vec{v}_{\otimes}\right)=-\partial \rho / \partial t \text { (continuity equation), } \\
& \omega=m \partial S / \partial t-\frac{m}{2} \vec{v}_{\otimes},
\end{aligned}
$$

which is equivalent to

$$
m D \overrightarrow{\mathrm{v}}_{\otimes} / D t=-\vec{\nabla} \omega(\text { Euler equation), }
$$

and

$$
[H-\omega] \Phi=\epsilon \Phi \text { (equation of state) . }
$$

The three equations are (a) the continuity equation [Eq. (5)], (b) a dynamical equation [Eq. (6)] describing the acceleration of a given mass probability element analogous to the Euler equation in fluid dynamics, and (c) a modified Schrödinger equation of state [Eq. (7)] which

\footnotetext{
${ }^{4}$ If recent suggestions that the motion of the nuclear shape may be dissipation dominated (Swiatecki, 1972; Myers, 1976) are borne out, then the importance of inertial parameters in the dynamical process could be substantially reduced.

${ }^{5}$ Some of this research has been partially published or reported earlier. See Griffin and Kan (1974, 1975); Kan (1975); Kan and Griffin $(1974 a, 1974 b, 1976)$.
}

has a form similar to a stationary state Schrödinger equation, and serves to relate the density of the Schrödinger fluid (given by the local Schrödinger probability density, $|\Phi|^{2}$ ) with the properties of the system which are incorporated into the Hamiltonian operator, as modified by the fluid dynamical properties, through the addition of the "dynamical modification potential" $-\omega$.

This derivation already suffices to sustain the claim that Schrödinger's equation, even at the most elementary one-particle level, is already naturally capable of reinterpretation ${ }^{6}$ in fluid dynamical terms (Madelung, 1926). Consequently any fluid dynamical theory of many-body systems described by the Schrödinger equation ought to exploit and build upon this natural structural relationship between the time-dependent quantum theory and the classical fluid theory.

\section{B. Dynamical compressibility}

We turn now to the question of dynamical compressibility of the Schrödinger fluid, and emphasize the fact that a flow is incompressible only when the total time derivative of the density vanishes; i.e., when

$$
D \rho / D t=\partial \rho / \partial t+\overrightarrow{\mathrm{v}}_{\otimes} \cdot \vec{\nabla} \rho=0 .
$$

[Note that in combination with the continuity equation (5), (8) is equivalent to the requirement that the divergence of the velocity field vanish, $\vec{\nabla} \cdot \vec{v}_{\otimes}=0$ whereever $\rho \neq 0$.] More specifically, the notion of static incompressibility, which simply states that the volume occupied by the total nuclear mass remains constant, is not sufficient to guarantee that the dynamical flow in nuclei is incompressible. In particular, a given amount of matter may be contained in a given volume and that volume may change its shape in such a way that the average density over the volume remains constant; still the flow pattern may be compressible, since small density ripples in the system may flow from place to place resulting in local variations from regions where the density is increasing (compression) to other regions where the density is decreasing.

\section{The effect of compressible flow on inertial parameters}

Indeed the whole purpose of this discussion is to emphasize the fact that such propagation of density ripples from place to place is a dominant effect in the vibrational inertia which one calculates from the cranking model (Inglis, 1954; Belyaev, 1959). Figure 6 demonstrates this statement more specifically in schematic terms (Griffin and Kan, 1974). It exhibits a level crossing of two single-particle levels in which the particle adiabatically makes an adjustment from orbit " $a$ " to orbit " $b$ ", and the uppermost level remains unfilled both to the left and to the right of the crossing. This is the expected behavior when the collective motion is very slow.

\footnotetext{
${ }^{6}$ E. Madelung (1926) first suggested a fluid dynamical interpretation of this type, but it does not seem to have been utilized effectively to describe actual physical processes, perhaps because atoms, being dominated by a fixed central Coulomb field, are less fluid-like than nuclei.
} 


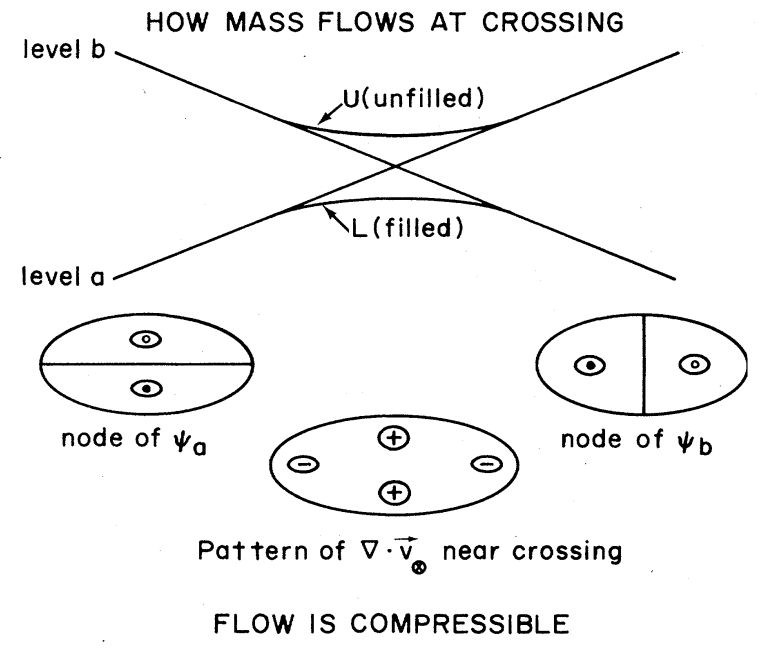

FIG. 6. Adiabatic adjustment to the lower state $\Psi_{L}$ at a crossing leads to compressible flow of nuclear matter.

We have schematized the levels " $a$ " and " $b$ " as singlenode states, " $a$ " having a node along the $x$ axis, and " $b$ " a node along the $y$ axis. When the wave function adjusts by remaining in the lower filled level then the mass flow pattern near the crossing is a compressible flow pattern: the divergence of the irrotational velocity field is represented schematically in the ellipse at the center of Fig. 8, where the sign of the divergence is positive in the region where level " $a$ " has a maximum and level " $b$ " has a node, and negative where the level " $a$ " has a node and level " $b$ " has a maximum. Thus one can see that when particles are adjusting to crossings of levels at the top of the Fermi sea, then the flow is compressible.

Just such compressible flow at level crossing (Griffin and Kan, 1974) is of overwhelming importance for the inertial parameters associated with nuclear shape vibrations. When one estimates (Griffin, 1969b; Primack, 1966) on the basis of the cranking model just how much such crossings contribute to the inertial parameters for quadrupole deformation, the contribution on the average is 1000 times the value of the inertia for incompressible irrotational flow when the particles are taken as completely independent particles, and still ten times that value (Griffin, 1971) for systems paired with reasonable pairing strengths.

Thus one concludes that in either case the effects of compressibility on collective nuclear inertia are substantial and are dominated by the rearrangement of the last few nucleons. A natural corollary is that, since in each nucleus the inertial parameters will be strongly affected by the details of level crossings near the Fermi sea, then the inertial parameter for each nucleus is a unique function specifically associated with the particular structure for its own values of $(N, Z)$. It therefore follows that when the nuclear flow is finally subjected to a complete dynamical analysis, one must expect at the most detailed level to treat each nucleus as separate and distinct. Then indeed each of the 2000 nuclei in the periodic table must ultimately be considered a distinct dynamical fluid.

\section{Analogy with Strutinsky shells}

The analogy between this inertial effect of compressibility and Strutinsky's (Strutinsky, 1968; Brack et al., 1972) generalized shell correction for potential energies is close. Both effects arise from the properties of a few nucleons in levels near the top of the Fermi sea. In the Strutinsky-potential-energy case, the crucial question is whether those levels are closer together or further apart than the smoothed average level density. ${ }^{7}$

For inertial parameters the question is instead whether the level crossings occur more frequently or less frequently. In both cases one obtains for each nucleus at each shape a specific correction to the collective potential energy on the one hand or to the inertial parameters (collective kinetic energy) on the other. Thus whether nuclei are viewed as static objects, whose collective properties at any shape require knowledge only of a potential energy, or as dynamical objects, whose inertial parameters are also crucial for the description of their motions, one comes to the conclusion that each nucleus is a separate and unique object exhibiting its own specific and distinct finite-particle-number properties. These, of course, are expected to diminish, relatively, in importance as the number of particles becomes very large. But for nuclei they still exert a significant influence.

\section{E. Every nucleus a fluid of its own}

The conclusion then emerges that nuclei offer a unique testing ground for the ultimate theories of fluid dynamics for two reasons: (a) that nuclei promise to be the first many-body systems which will be microscopically analyzed from first principles and (b) that since nuclei do exhibit these distinctly finite-particle-number effects, each offers its own specific test for a dynamical theory. Thus altogether nuclei may offer a much richer field for testing fluid dynamical theories than do systems with infinitely many particles, where no such idiosyncratic properties are expected to demand explanation.

\section{NOVEL VIEW OF QUANTUM PHYSICS VIA THE SINGLE-PARTICLE SCHRÖDINGER FLUID}

In this third part, we exhibit some grounds for expecting that the single-particle Schrödinger fluid (Kan, 1975; Kan and Griffin, 1976) can suggest useful new physical characterizations of time-dependent quantal systems. Specifically, velocity fields arise naturally therefrom, and lead, in turn, to novel expressions for such physical quantities as collective kinetic energy, etc. We believe that the importance of this lies not in the several results already obtained, but in the promise that new expressions, from a new viewpoint, for basic physical quantities, can allow us the opportunity to perceive relevant new insights which, although complicated in the old language, might be substantially simplified in the new.

Indeed, the fluid dynamical interpretation of quantal systems has already led to at least one spinoff idea not

\footnotetext{
${ }^{7}$ For a schematic discussion of Strutinsky shell corrections, see Griffin (1969a).
} 
immediately connected with the context in which it first arose: the nonlinear Schrödinger equation for friction (Kan and Griffin, 1974a, b). This description arises naturally when one considers the fluid dynamical interpretation of one-dimensional problem. We shall discuss it in more detail in Sec. IV.

\section{A. Irrotational velocity}

Already in Eq. (4) we find the first velocity field given by the gradient of the phase, $\vec{v}_{\otimes}=-\vec{\nabla} S$. This field inevitably arises in the problem of the single-particle Schrödinger fluid. Since it is a gradient almost everywhere, its curl is almost everywhere zero, and we refer to it as the irrotational velocity field, $\vec{v}_{\otimes}$. It leads naturally to a current given by the density times that irrotational velocity field, $\vec{J}=\rho \vec{v}_{\otimes}$, which current obeys the continuity equation.

One especially interesting property of this irrotational velocity field $\vec{v}_{\otimes}$ (which, we emphasize, is uniquely prescribed once the wave function which solves the Schrödinger equation is known) is that it is not so simple as one at first thinks, because it may exhibit line vortices wherever the wave function has a zero. Thus, if the solution of the time-dependent Schrödinger equation vanishes somewhere, then singularities in the velocity potential (which itself is not a physically observable quantity) may occur there. These singularities violate no physical principles, but do allow a rich structure to exist in the irrotational velocity fields which occur in the single-particle Schrödinger fluid.

\section{B. Regular velocity}

In addition to the irrotational velocity field, which alone is uniquely prescribed by the exact wave function, other velocity fields, which solve the continuity equation under various selected conditions (Kan, 1975; Kan and Griffin, 1976) also can be introduced naturally into the time-dependent description of the single-particle Schrödinger fluid. One such field is the "regular velocity field" $\overrightarrow{\mathrm{v}}_{R}$, which is prescribed to solve the continuity equation but to have no singularity in the finite space i.e.,

$$
\rho \vec{\nabla} \cdot \overrightarrow{\mathrm{v}}_{R}+\overrightarrow{\mathrm{v}}_{R} \cdot \vec{\nabla} \rho=-\partial \rho / \partial t .
$$

As an example, for pure rotations the regular velocity field is simply

$$
\overrightarrow{\mathrm{v}}_{R}=\vec{\Omega} \times \overrightarrow{\mathrm{r}} \text {, }
$$

where $\vec{\Omega}$ is the rotational angular velocity vector, and $\vec{r}$ is the position vector. This regular field is just the familiar velocity field for rigid-body rotation about an axis. In this case, it is obviously regular everwhere. (It is also, incidentally, an incompressible flow field in this case.) Other useful velocity fields are discussed in Kan (1975) and Kan and Griffin (1976).

\section{Collective kinetic energy}

We mention these two particular velocity fields just to be able to exhibit the fact that the collective kinetic energy of the single-particle Schrödinger fluid assumes an especially simple form (Kan, 1975; Kan and Griffin,
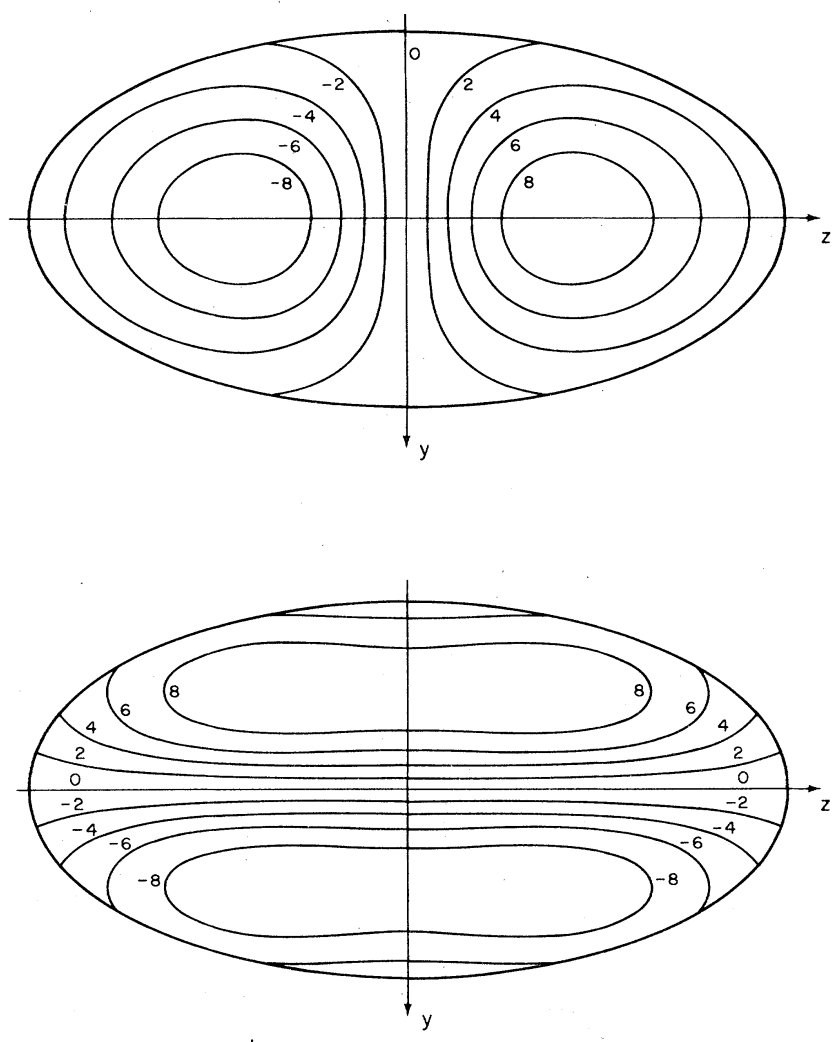

FIG. 7. This figure, together with several subsequent figures $(8,12,16)$ attempts to portray in the fluid dynamical imagery (Kan, 1975; Kan and Griffin, 1976) of the wave functions $\Psi=u$ $+i \Omega v$ for single particles in a rotating, deformed simple harmonic oscillator potential. Here $u$ and $v$ are real, and the lines are equiamplitude contours for a particle in the $\left(N_{x}, N_{y}, N_{z}\right)=(0,0,1)$ state. The upper figure portrays $u$; the lower, $v$. In this and subsequent figures (8-16), the contour values shown are in arbitrary relative units.

1976) when one rewrites it in terms of these two vellocity fields, $\overrightarrow{\mathrm{v}}_{\otimes}$ and $\overrightarrow{\mathrm{v}}_{R}$. The general expression for the kinetic energy is

$$
K_{\mathrm{col1}} \equiv \int \rho(\partial S / \partial t) d \tau
$$

After some algebraic manipulation, one obtains the result

$$
K_{\mathrm{col1}}=\frac{1}{2} \int \rho \overrightarrow{\mathrm{v}}_{\otimes} \cdot \overrightarrow{\mathrm{v}}_{R} d \tau \text { in general }
$$

through terms quadratic in $\dot{\alpha}$. Thus the kinetic energy is described simply by a volume integral of the densityweighted scalar product of the irrotational with the regular velocity field. [Note that the result (12) is identical with the corresponding single-particle contribution to the cranking model (Inglis, 1954; Belyaev, 1959) inertia.] For rotations, the simple form (12) becomes even more intriguing because of Eq. (10)

$$
K_{\text {rot }}=\frac{1}{2} \int \rho \overrightarrow{\mathrm{v}}_{\otimes} \cdot(\vec{\Omega} \times \overrightarrow{\mathrm{r}}) d \tau .
$$

The hope of connecting Eq. (13) with the well-known fact that large (Amado and Brueckner, 1959), smooth 

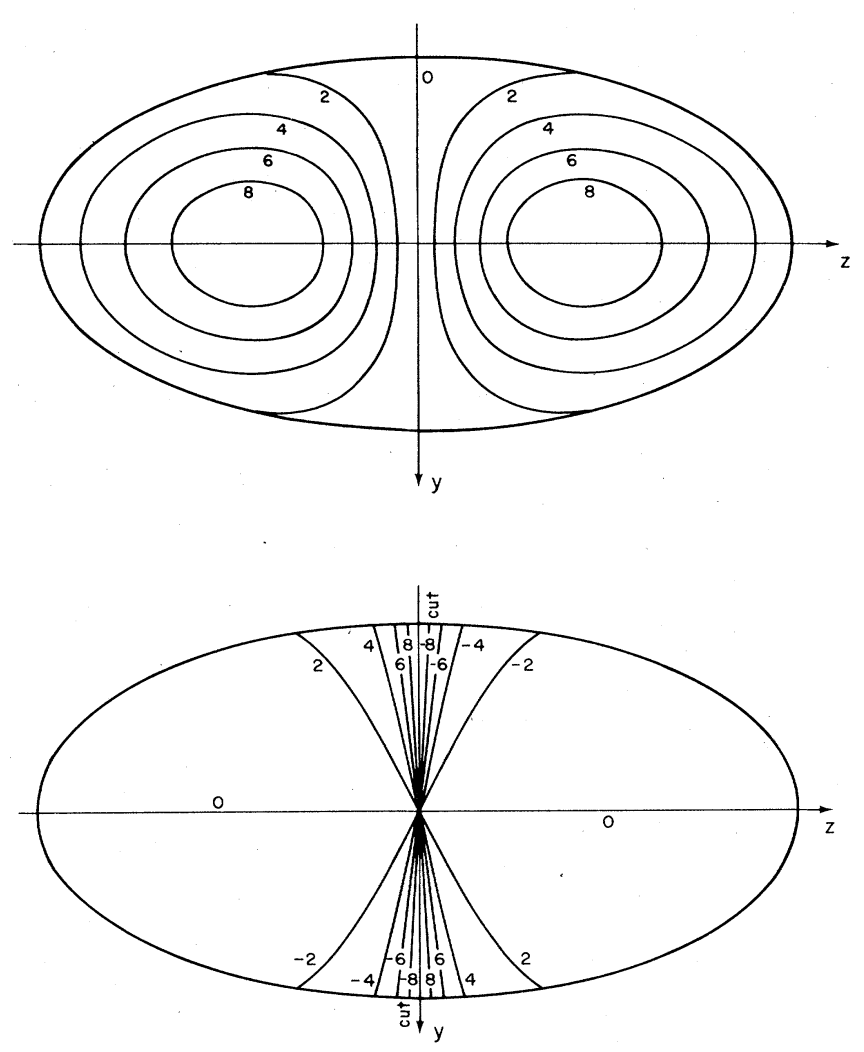

FIG. 8. For a slow rotation of the potential ( $\Omega$ small), the magnitude $\Phi$, and phase $S$, of the wave function $\Psi$, of Fig. 7 are displayed, in the upper and lower figures, respectively. Note that the lower figure exhibits a phase $S$ which, as a velocity potential, describes a velocity field $\vec{v}_{\otimes}=-\vec{\nabla} S$ with streamlines encircling the $x$ axis (the only line vortex in this field) in a counterclockwise direction.

(Migdal, 1959), or special (Bohr and Mottelson, 1955) systems rotate with rigid-body kinetic energies

$$
T=\int \rho(\vec{\Omega} \times \overrightarrow{\mathrm{r}})^{2} d \tau
$$

is irresistible.

Thus one finds for the kinetic energy a remarkably

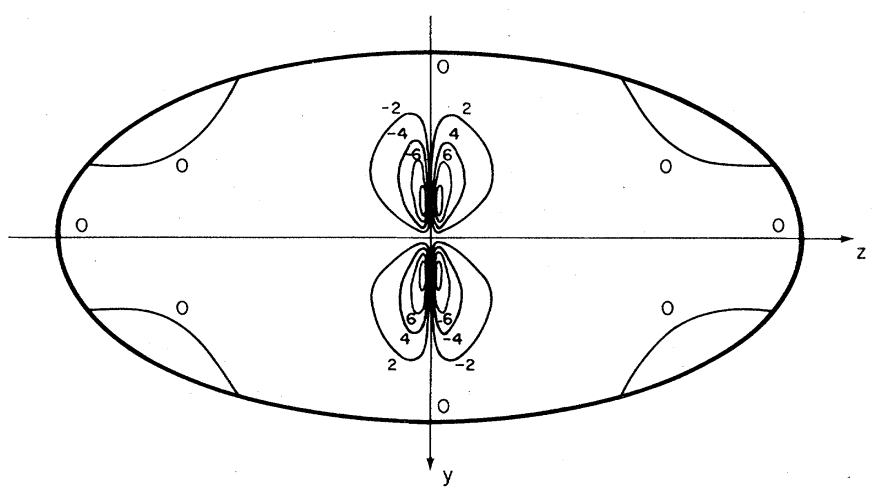

FIG. 9. The divergence $\vec{\nabla} \cdot \vec{v}=-\vec{\nabla}^{2} S$, weighted by $\rho$, of the irrotational velocity field defined by the phase $S$ in Fig. 8, is shown as a function of position. Since a nonzero divergence implies compressible flow [(by Eq. (8)], we refer to the quantity $-\vec{\nabla} \cdot \vec{v}_{\otimes}$ as the "local compression."

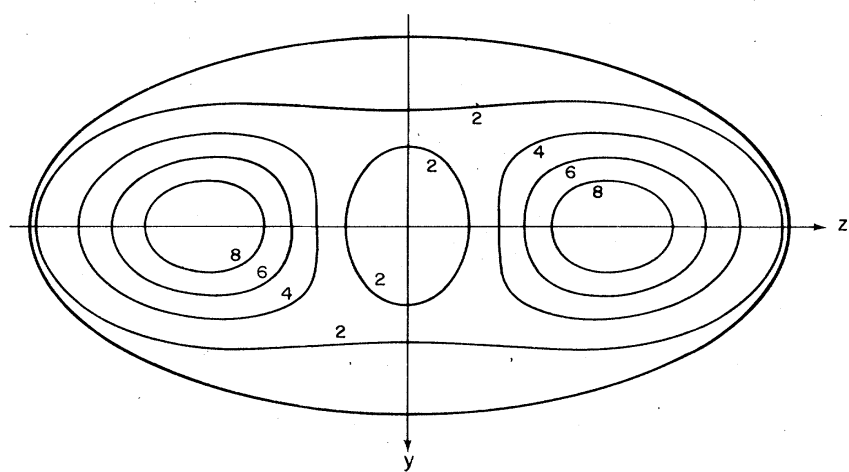

FIG. 10. The collective kinetic energy density $\rho \vec{v}_{\otimes} \cdot(\vec{\Omega} \times \overrightarrow{\mathrm{r}})$ of Eq. (10) in text, is shown as a function of position for the slow rotation of Fig. 8 and 9.

simple and symmetric quadratic form involving, not, as in the classical case, the square of one single velocity, but instead the scalar product between two distinct velocities, each of which arises naturally out of the description. Although the physical content of Eq. (12) goes no further than the cranking model so long used in nuclear structure physics, one hopes still that it offers an opportunity for new insight and development.

\section{Single-particle Schrödinger fluid: Some details}

Several figures, 7-17, exhibit calculations of some of the fluid dynamical properties of simple singleparticle Schrödinger fluids: the real and imaginary parts (Fig. 7), the magnitude and phase of the wave function $\Psi$ (Fig. 8), the local decompression, $\vec{\nabla} \cdot \vec{v}_{\otimes}$ (Fig. $9)$, the collective kinetic energy density, $\rho \overrightarrow{\mathrm{v}}_{\otimes} \cdot(\vec{\Omega} \times \overrightarrow{\mathrm{r}})$ (Fig. 10), and the dynamical modification potential, $-\omega$ $=-m\left(\partial S / \partial t-\frac{1}{2} \vec{v}_{\otimes}^{2}\right)$. (Fig. 11) which occurs in the modified Schrödinger equation of state, Eq. (7), and whose gradient provides the accelerative force in the Euler equation (6b), are all shown ${ }^{8}$ for the rotation of the simplest nontrivial simple harmonic oscillator (SHO) wave

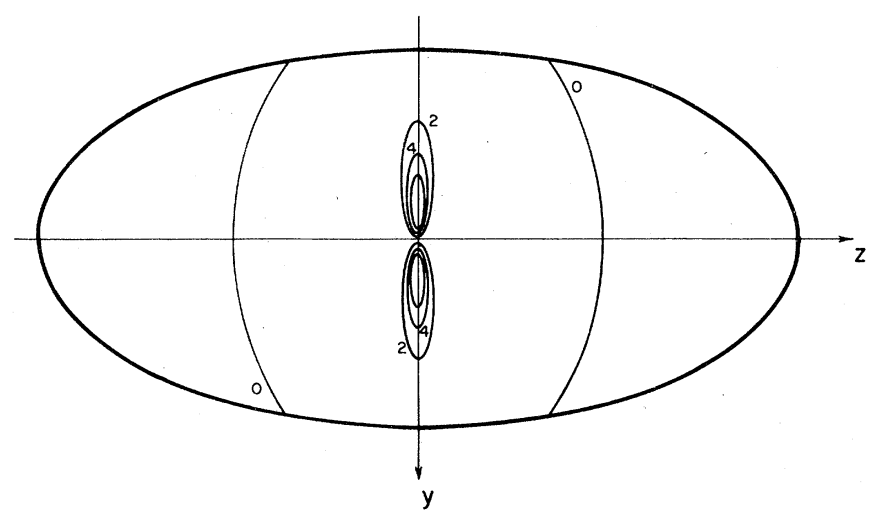

FIG. 11. The "dynamical modification potential," $-\omega=-m[(\partial S /$ $\partial t)-\frac{1}{2} \vec{v}_{\otimes}^{2}$ l, of Eq. (7) in text, weighted by $\rho$, is shown for the slow rotation of Figs. 8 and 9 .

${ }^{8}$ The local decompression, $\vec{\nabla} \cdot \vec{v}_{\otimes}$, and the dynamical modification potential, $-\omega$, shown in Figs. 9, 11, 13, and 15 are weighted by the density, $\rho$, in order to remove the singularities in these quantities. 

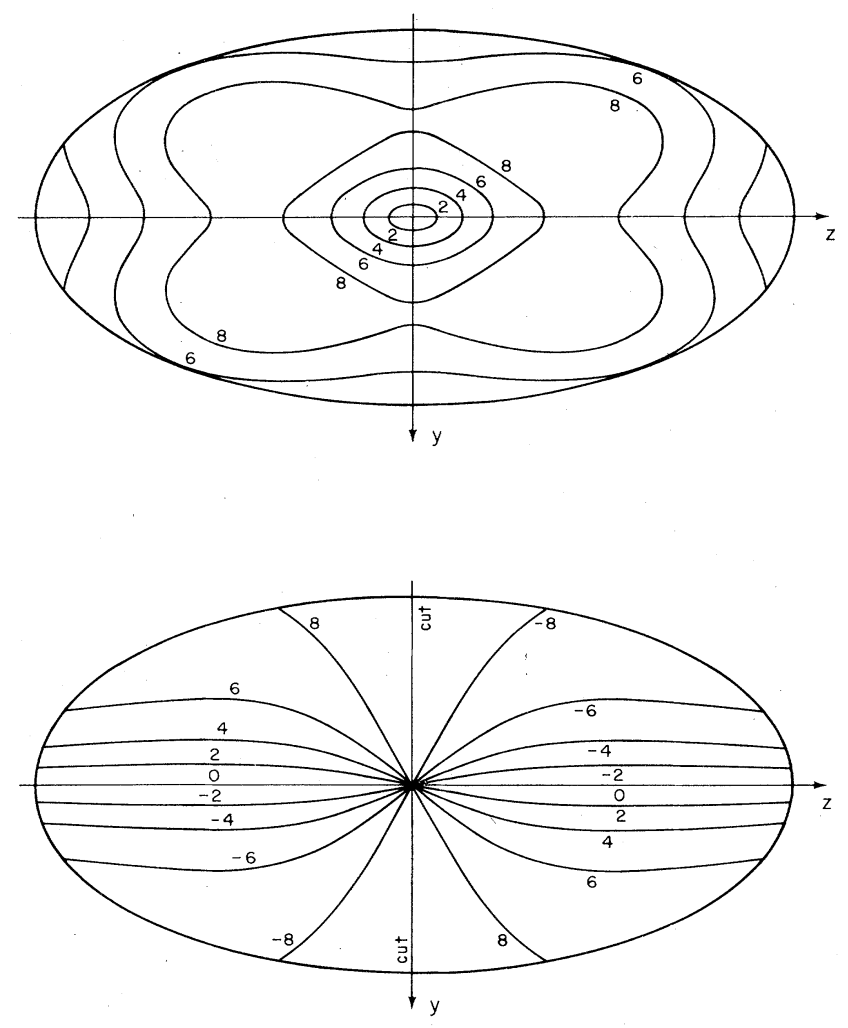

FIG. 12. The magnitude $\Phi$ (upper figures) and the phase $S$ (lower) are shown for a more rapidly rotating $\left(N_{x}, N_{y}, N_{z}\right)$ $=(0,0,1)$ function. Here $\Omega$ is chosen so that the imaginary part, $i \Omega v$ of $\Psi$ is of comparable average magnitude with the real part, $u$ in $\Psi=u+i \Omega v$. The velocity field, $\overrightarrow{\mathrm{v}}_{\otimes}=-\vec{\nabla} S$, here will also exhibit closed trajectories counterclockwise around the $x$ axis, but with a more nearly uniform velocity than in the slow rotation of the same wave function (Fig. 8). (We note that this figure should be considered as illustrative only, since the result of the cranking model perturbation treatment used here may differ substantially from the exact solution when $\Omega$ becomes large.)

function $\left(N_{x}, N_{y}, N_{z}\right)=(0,0,1)$. Figures $12-15$ exhibit the same quantities as in Figs. 8-11, but for a faster rotation.

In addition Figs. 16 and 17 exhibit the velocity potential $S$ and the corresponding streamlines for a some-

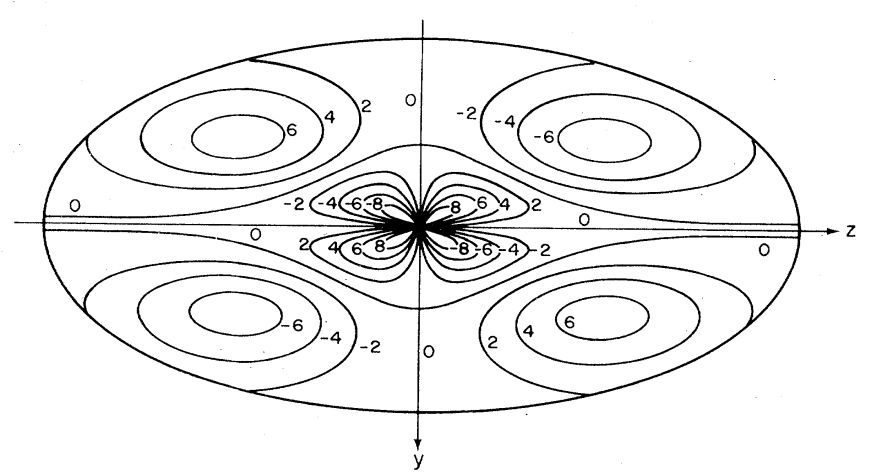

FIG. 13. The "local decompression," $\vec{\nabla} \cdot \vec{v}_{\otimes}=-\vec{\nabla}^{2} S$, weighted by $\rho$, is displayed for the rapid rotation of Fig. 10 .

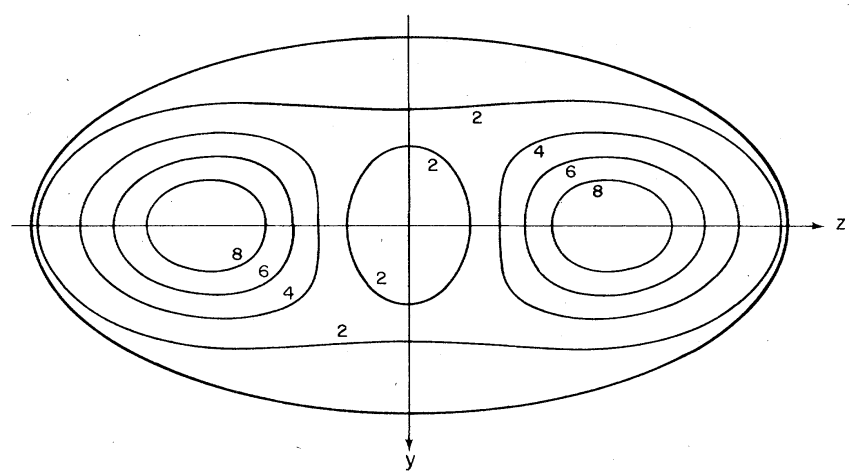

FIG. 14. The collective kinetic energy density, $\rho \overrightarrow{\mathrm{v}}_{\otimes} \cdot(\vec{\Omega} \times \overrightarrow{\mathrm{r}})$, is shown as a function of position for the rapid rotation of Figs. 12 and 13 .

what more complicated SHO wave function, $\left(N_{x}, N_{y}, N_{z}\right)$ $=(1,1,2)$. Especially these latter two figures, we feel, offer a glimpse into the marvelous and subtle world of microscopic quantum dynamics in which the complete theory of dynamical processes is ultimately to be constructed. Further details are cited in the figure captions.

\section{QUANTUM FRICTION-ONE SPINOFF}

Finally, we offer an example to show that when a new intuitional viewpoint is found, spinoff ideas may occur which affect other problems in an unexpected way. Out of the work on the single-particle Schrödinger fluid there arose a simple way to construct a quantum description of friction.

This problem had been under discussion in the nuclear community for a few years, especially at Berkeley, where conversations were shared with W. Swiatecki and G. Süssman on the subject. We wondered among ourselves how a system which is oscillating in one dimension and gradually slowing down because of friction is to be described in the quantum realm. One naturally thinks of describing the system in terms of an imaginary potential, but quickly realizes that an imaginary potential describes a system of diminishing probability rather than the desired diminishing of dispacement amplitude. In contrast to the solution in terms of an

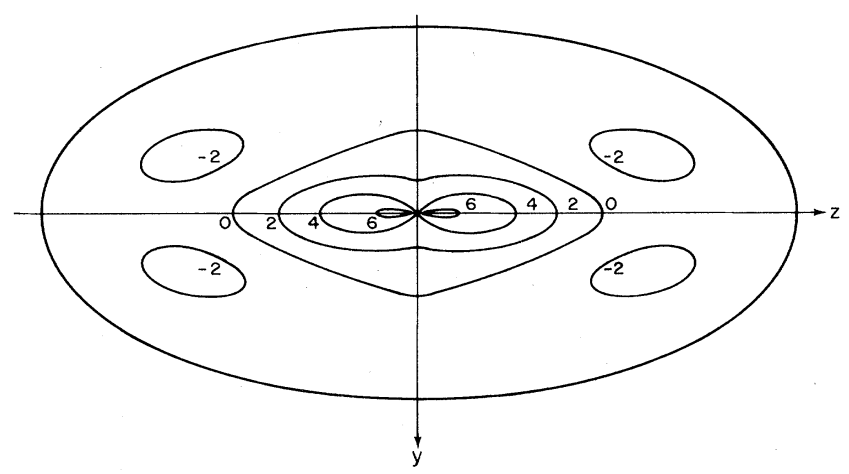

FIG. 15. The dynamical modification potential, $-\omega=-m[(2 S)$ $\left.\partial t)-\frac{1}{2} \overrightarrow{\mathrm{v}}_{\otimes}^{2}\right]$, weighted by $\rho$, is shown for the rapid rotation of Figs. 12 and 13 . 


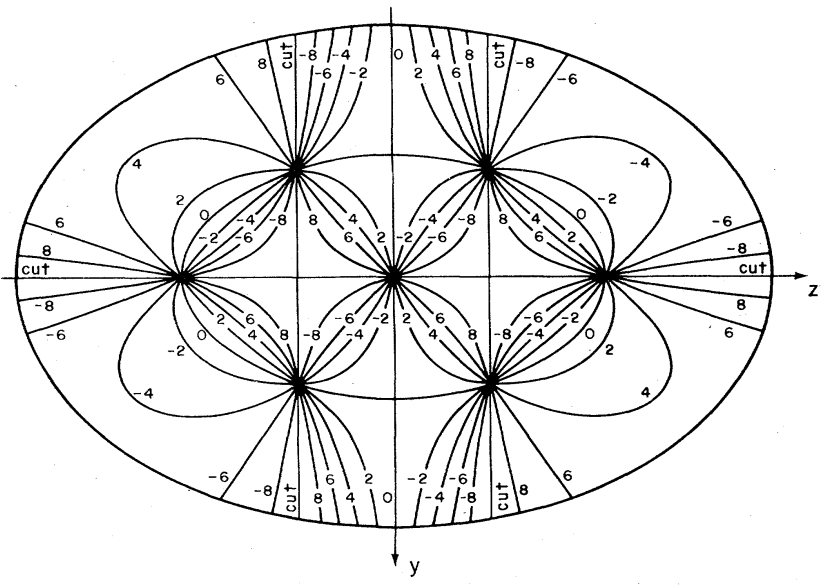

FIG. 16. The phase, or velocity potential $S$ in $\Psi=\Phi \exp [-(\mathrm{im} /$ $\hbar) S$ ], is displayed for a more complicated single-particle function $\left(N_{x}, N_{y}, N_{z}\right)=(1,1,2)$ than that of Figs. $7-15$. In this case seven line vortices occur, as compared to one in the $\left(N_{x}, N_{y}, N_{z}\right)=(0,0,1)$ case.

imaginary potential, one needs instead a wave function whose probability integral is constant but which describes a motion, the magnitude of whose oscillations diminishes with time.

Some time after this fluid interpretation had been developed, we realized that the velocity potential offered an opportunity to solve this problem very simply (Kan and Griffin, 1974a,b). We here briefly summarize the solution, which leads to a nonlinear equation similar to that which Kostin (1972) had discovered earlier in studying descriptions of Brownian motion by quantal Langevin equations.

Consider a one-particle Hamiltonian $H_{0}$. Upon addition of a constant multiple of the velocity potential, $S$, to the one-particle Hamiltonian $H_{0}$, one insures that in the classical limit (where gradients of the potential term in the Hamiltonian define the forces) he obtains a classical force proportional to the negative gradient of

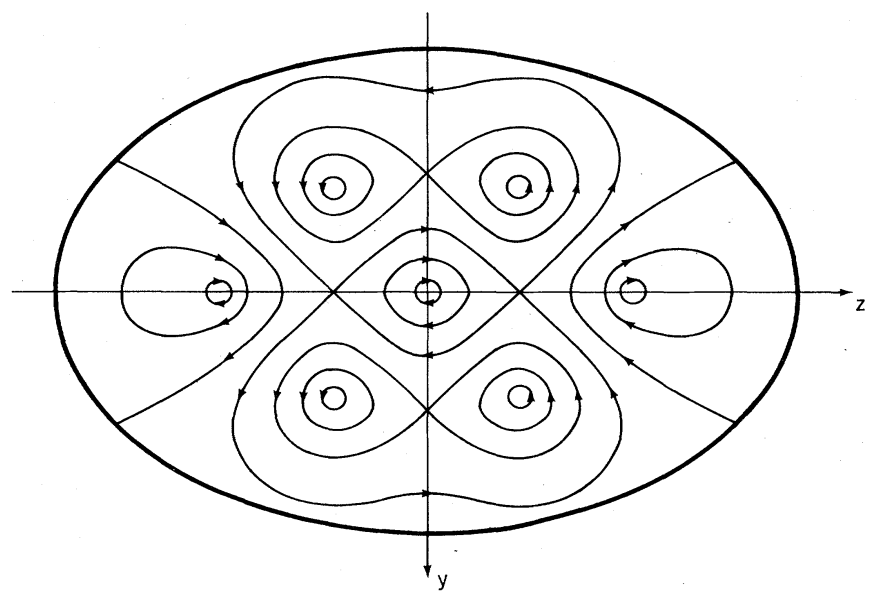

FIG. 17. The streamline pattern implied for $\vec{v}$ by the velocity potential of Fig. 16 is displayed. It is remarkable how intricate it is, even for a pure rotation of such a simple, $\left(N_{x}, N_{y}, N_{z}\right)=(1,1,2)$, single-particle wave function. the irrotational velocity potential for the wave function. That is,

$$
H^{\prime}=H_{0}+\eta S
$$

implies that in the classical limit the potential forces $\overrightarrow{\mathrm{F}}_{p}$ will be augmented by a frictional force $\overrightarrow{\mathrm{F}}_{f}$

$$
\overrightarrow{\mathrm{F}}=\overrightarrow{\mathrm{F}}_{p}+\overrightarrow{\mathrm{F}}_{f},
$$

where

$$
\overrightarrow{\mathrm{F}}_{f}=\eta \vec{\nabla} S=-\eta \overrightarrow{\mathrm{v}}_{\otimes}
$$

is proportional to the negative velocity of the particle.

Of course, when a wave packet is being used to describe a localized, classical particle, such a force is proportional to the negative velocity of the particle itself, as required by the correspondence principle. Thus addition of the velocity potential to a Hamiltonian guarantees in a classical limit exactly the kind of frictional force desired.

The second step in constructing the equation is then to add a counterterm to cancel the expectation value of the velocity potential (which, after all, is not to be considered physically as an energy) in order not to alter the usual relationship between the expectation value of $H$ and the expectation value of $T+V$. Then one has for the Hamiltonian

$$
H=H_{0}+\eta S-\eta\langle S\rangle
$$

which, ${ }^{9}$ by construction, guarantees that

$$
\langle H\rangle=\left\langle H_{0}\right\rangle=\langle T+V\rangle \text {. }
$$

The resulting nonlinear time-dependent Schrödinger equation is

$$
i \hbar \stackrel{\circ}{\Psi}=H \Psi=\left[H_{0}+(\eta \hbar / 2 i m)\left(\ln \Psi / \Psi^{*}-\left\langle\ln \Psi / \Psi^{*}\right\rangle\right)\right] \Psi .
$$

The equation is remarkable in that, although it is a nonlinear equation, it does not violate probability conservation, as in general one must expect nonlinear equations to do. The reason: Its nonlinear term depends only on the phase, and not on the magnitude, of $\Psi$. Moreover, for the case of a zero-order Hamiltonian which describes a particle in a simple harmonic oscillator well, this wave equation exhibits exact, closedform solutions

$$
\Psi=\psi_{n}[x-X(t)] \exp i\left\{x P(t) / \hbar-g_{n}(t)\right\},
$$

where $\psi_{n}(x)$ is any eigenfunction of $H_{0}$, and

$$
\begin{aligned}
& X(t)=X_{0}[\exp (-\gamma t)] \cos (\omega t-\delta), \\
& P(t)=-m X_{0}[\exp (-\gamma t)][\omega \sin (\omega t-\delta)+\gamma \cos (\omega t-\delta)] \\
& g_{n}(t)=\left(n+\frac{1}{2}\right) \omega_{0} t+\int_{0}^{t} \frac{d t^{\prime}}{\hbar}\left[\frac{P^{2}}{2 m}-\frac{m \omega_{0}^{2}}{2} X^{2}-\frac{\eta}{m} P X\right]
\end{aligned}
$$

\footnotetext{
${ }^{9}$ We note that the use of the word "Hamiltonian" as applied to an object like $H$ in Eq. (18) is worth some discussion (Amiot and Griffin, 1975). One can say for nonlinear problems like the present one at least that each operational implication of such a name ought to be inspected closely before utilization.
} 
These describe the damped oscillations of wave packets whose internal structure may correspond to the amplitude function of any eigenstate of the undamped simple harmonic oscillator Hamiltonian $H_{0}$. The oscillation frequency is shifted slightly from the oscillator frequency $\omega_{0}$, exactly to the value which applies to the classical damped oscillator

$$
\omega^{2}=\omega_{0}^{2}-\gamma^{2}
$$

where

$$
\gamma=\eta / 2 m \text {. }
$$

Each of these solutions develops in time ultimately into an eigenstate of the undamped Hamiltonian. Moreover, for such an eigenstate, the complex phase is constant in space, so that the nonlinear frictional force vanishes identically.

Thus this equation resolves in a very simple way the question of whether such a frictional term ought or ought not to operate on the zero-point velocities, which are still finite in the quantum ground state. This particular nonlinear theory answers that question unambiguously: Frictional forces do not apply to the zeropoint oscillations of the particle, nor, indeed, to any velocity not associated with a nonzero matter current $\vec{J}$. This answer is in our judgement an encouraging and agreeable result for two reasons.

The first is the inescapable implication of the theory that undamped solutions exist, at least in principle, even for arbitrarily high excitation energies. At first we considered this a puzzling and disturbing result. But when viewed from the background of the many-body problem out of which the quantum time-dependent frictional equation (20), obtained by inserting (18) into the time-dependent Schrödinger equation, purports to describe just one degree of freedom, these excited stationary states find a natural role as reflections in the reduced subspace, of the normal mode eigen-oscillations of the many-body system. They assert the very plausible claim that, if the many-body system is carefully prepared at $t=0$ to be in a well-defined stationary excited state (i.e., in a normal mode), then the projection of that state onto the one-dimensional subspace described by Eq. (20) will also oscillate without damping. Of course, the spectra of such stationary states will hardly be so simple as that of the present pure oscillator model. ${ }^{10}$ Nevertheless, a plausible physical content seems to exist in these unanticipated stationary solutions of Eq. (20).

In addition, the vanishing of frictional forces in stationary states resolves certain paradoxical implications of alternative descriptions, especially the occurrence of solutions which, as $t \rightarrow \infty$, grow narrower and narrower in the physical momentum space (Kanai, 1948;

\footnotetext{
${ }^{10}$ One might reasonably conjecture more: That the "best" solution to such a reduction problem may not be universal, but may vary with the properties of the underlying many-body system. Then many different "theories" of quantal friction might be "correct." Hasse (1975a) lists three possible one-dimensional forms for nonlinear frictional Hamiltonians which he (1975b) and others (Süssman, 1974; Albrecht, 1975) have studied.
}

Britten, 1950; Bopp, 1962). We note that this agreeable feature of Eq. (21) is essentially connected with its nonlinearity; as the state approaches a stationary state, the frictional force is able to diminish to zero only because of its dependence upon $\Psi$.

Having such a description for the one-dimensional friction problem, one naturally wonders whether it can be applied to nuclear problems ${ }^{11}$ and generalized to the more complicated problem of describing the processes of nuclear viscosity. The answer to this question is open, but our attitude toward it is optimistic.

\section{ACKNOWLEDGMENTS}

The authors wish to acknowledge with gratitude the innumerable conversations we have enjoyed with so many colleagues about the topics discussed here, but especially with Dr. C. Y. Wong. We wish also to express our gratitude to Dr. G. T. Seaborg and his collaborators and to Dr. J. Wilczynski for their permission to reproduce here figures from their published work.

\section{REFERENCES}

Adam, J. R., M. R. Lindblad, and C. D. Hendricks, 1968, J. Appl. Phys. 39, 5173.

Albrecht, K., 1975, Phys. Lett. B 56, 127.

Amado, R. D., and K. A. Brueckner, 1959, Phys. Rev. 115, 778 .

Amiot, P., and J. J. Griffin, 1975, Ann. Phys. 95, 295.

Artukh, A. G., G. F. Gridrev, V. L. Mikheev, V. V. Volkov, and J. Wilczynski, 1973, Nucl. Phys. A 215, 91.

Belyaev, S. T., 1959, K. Dan. Vidensk. Selsk. Mat.-Fys. Medd. 31 , no. 11.

Bohr, A., and B. R. Mottelson, 1955, K. Dan. Vidensk. Selsk. Mat.-Fys. Medd. 30, no. 1 .

Bopp, F., 1962, Z. Angew. Phys. 14, 699.

Brack, M., J. Damgaard, A. S. Jensen, H. C. Pauli, V. M. Strutinsky, and C. Y. Wong, 1972, Rev. Mod. Phys. 44, 320. Britten, W. E., 1950, Phys. Rev. 77, 396.

Griffin, J. J., 1969a, Comments Nucl. Part. Phys. III, 58. Griffin, J. J., 1969b, in Proceedings of the Second International Atomic Energy Agency Symposium on Physics and Chemistry of Fission, Vienna, Austria, 1969 (IAEA, Vienna), p. 3 .

Griffin, J. J., 1971, Nucl. Phys. A 170, 395.

Griffin, J. J., and K.-K. Kan, 1974, in Proceedings of the Third International Atomic Energy Agency Symposium on Physics and Chemistry of Fission, Rochester, New York, 1973 (IAEA, Vienna), Vol. 1, p. 533.

Griffin, J. J., and K.-K. Kan, 1975, in Proceedings of the Third International Workshop on Gross Properties of $\mathrm{Nu}$ clei and Nuclear Excitations, Hirschegg, Kleinwal sertal, Austria, 1975, edited by W. J. Myers (Inst. f. Kernphysik, Technische Hochschule Darmstadt, Germany, Rept. No. AED-Conf-75-009-000), p. 56 .

Griffin, J. J., and C. Y. Wong, 1975, Bull. Am. Phys. Soc. II 20, 1158; paper to be published.

Hasse, R. W., $1975 \mathrm{a}$, in Proceedings of the Third International Workshop on Gross Properties of Nuclei and Nuclear Excitations, Hirschegg, Kleinwalsertal, Austria, 1975, edited by W. J. Myers (Inst. f. Kernphysik, Technische Hochschule Darmstadt, Germany, Rept. No. AED-Conf-75-009-000), p. 26.

Hasse, R. W., 1975b, J. Math. Phys. 16, 2005. Huizenga, J. R., 1975, Nukleonika 20, 291; private communi-

\footnotetext{
${ }^{11}$ See Immele ét al. (1975) for a beginning in this direction.
} 
cation.

Immele, J. D., K.-K. Kan, and J. J. Griffin, 1975, Nucl.

Phys. A 241, 47.

Inglis, D. R., 1954, Phys. Rev. 96, 1059.

Kan, K.-K., and J. J. Griffin, 1974a, Phys. Lett. B 50, 241.

Kan, K.-K., and J. J. Griffin, 1974b, in Proceedings of the International Conference on Reactions between Complex Nuclei, Nashville, Tennessee, 1974, edited by R. L. Robinson, F. K. McGowan, J. B. Ball, and J. H. Hamilton (North-Holland, Amsterdam), Vol. 1, p. 127.

Kan, K.-K., 1975, Univ. of Maryland (College Park, Maryland) Ph.D. thesis, June, 1975.

Kan, K.-K., and J. J. Griffin, 1976, Univ. of Maryland Technical Report No. 76-086, and papers to be published.

Kanai, E., 1948, Prog. Theor. Phys. 3, 440.

Kostin, M. D., 1972, J. Chem. Phys. 57, 3589.
Kratz, J. V., A. E. Norris, and G. T. Seaborg, 1974, Phys. Rev. Lett. 33, 502 .

Madelung, E., 1926, Z. Phys. 40, 332.

Migdal, A. B., 1959, Nucl. Phys. 13, 655 (especially page 662).

Myers, W. J., 1976, in Proceedings of the Fourth Inter-

national Workshop on Gross Properties of Nuclei and Nuclear Excitations, Hirschegg, Austria, 1976 (Inst. f. Kernphysik,

Technische Hochschule Darmstadt, Germany).

Nörenberg, W., 1974, Phys. Lett. B 52, 289.

Nörenberg, W., 1975, Z. Phys. A 274, 241.

Primack, J. R., 1966, Phys. Rev. Lett. 17, 539.

Strutinsky, V. M., 1968, Nucl. Phys. A 122, 1.

Süssman, G., 1974, private communication cited in Hasse (1975a).

Swiatecki, W. J., 1972, private communication to J. J. G.

Wilczynski, J., 1973, Phys. Lett. B 47, 484. 